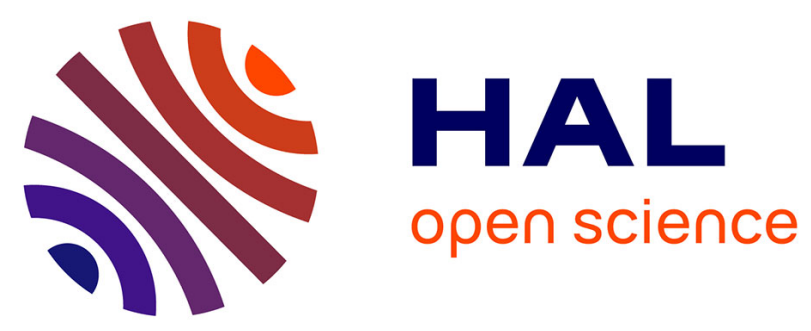

\title{
Formation Dynamics of Excited Neutral Nitrogen Molecules inside Femtosecond Laser Filaments
}

Rostyslav Danylo, Xiang Zhang, Zhengquan Fan, Dongjie Zhou, Qi Lü, Bin Zhou, Qingqing Liang, Songlin Zhuang, Aurélien Houard, André Mysyrowicz, et al.

\section{To cite this version:}

Rostyslav Danylo, Xiang Zhang, Zhengquan Fan, Dongjie Zhou, Qi Lü, et al.. Formation Dynamics of Excited Neutral Nitrogen Molecules inside Femtosecond Laser Filaments. Physical Review Letters, 2019, 123, pp.243203. 10.1103/PhysRevLett.123.243203 . hal-02414411

HAL Id: hal-02414411

https://hal-ensta-paris.archives-ouvertes.fr/hal-02414411

Submitted on 16 Dec 2019

HAL is a multi-disciplinary open access archive for the deposit and dissemination of scientific research documents, whether they are published or not. The documents may come from teaching and research institutions in France or abroad, or from public or private research centers.
L'archive ouverte pluridisciplinaire HAL, est destinée au dépôt et à la diffusion de documents scientifiques de niveau recherche, publiés ou non, émanant des établissements d'enseignement et de recherche français ou étrangers, des laboratoires publics ou privés. 


\title{
Formation Dynamics of Excited Neutral Nitrogen Molecules inside Femtosecond Laser Filaments
}

\author{
Rostyslav Danylo, ${ }^{1}$ Xiang Zhang, ${ }^{1}$ Zhengquan Fan, ${ }^{1}$ Dongjie Zhou, ${ }^{1}$ Qi Lu, ${ }^{1}$ Bin Zhou, ${ }^{1}$ Qingqing Liang, \\ Songlin Zhuang, ${ }^{1}$ Aurélien Houard $\odot,{ }^{2}$ André Mysyrowicz, ${ }^{2}$ Eduardo Oliva $\odot,{ }^{3,4, *}$ and Yi Liu $\odot^{1,2, \dagger}$ \\ ${ }^{1}$ Engineering Research Center of Optical Instrument and System, The Ministry of Education; \\ Shanghai Key Lab of Modern Optical System, University of Shanghai for Science and Technology, \\ 516, Jungong Road, 200093 Shanghai, China \\ ${ }^{2}$ Laboratoire d'Optique Appliquée, ENSTA Paris, Ecole Polytechnique, CNRS, Institut Polytechnique de Paris, \\ 828 Boulevard des Maréchaux, 91762 Palaiseau cedex, France \\ ${ }^{3}$ Departamento de Ingeniería Energética, ETSI Industriales, Universidad Politécnica de Madrid, E-28006 Madrid, Spain \\ ${ }^{4}$ Instituto de Fusión Nuclear "Guillermo Velarde", Universidad Politécnica de Madrid, 28006 Madrid, Spain
}

(Received 2 September 2019; published 12 December 2019)

\begin{abstract}
Nitrogen molecules are promoted to excited neutral states during femtosecond laser pulse filamentary propagation in atmosphere, leading to a characteristic UV fluorescence. Using a laser-induced fluorescence depletion technique, we measure the formation dynamics of these excited neutral nitrogen molecules with femtosecond time resolution. We find that the excited neutral molecules are formed in an unexpected ultrafast timescale of $\sim 4$ ps at 1 bar and $\sim 120$ ps at 30 mbar pressure. From this observation we deduce that the excitation of neutral $\mathrm{N}_{2}$ occurs via multiple collisions with hot free electrons. Numerical simulations based on rate equations reproduce well this ultrafast formation time and its dependence on gas pressure, and thus support this interpretation.
\end{abstract}

DOI: 10.1103/PhysRevLett.123.243203

A high power femtosecond laser pulse propagating in air undergoes filamentation. During this process, nitrogen molecules become ionized, resulting in a bright long plasma channel left in the wake of the filamentary laser pulse [1-3]. The density of this air plasma is in the range $10^{16}-10^{17} \mathrm{~cm}^{-3}$ and its length varies from a few centimeters to tens of meters or more, depending on the initial femtosecond pulse energy and beam convergence $[1,2,4]$. Several applications rely on the presence of this plasma channel. It can act as a terahertz or radio frequency radiation antenna [5-7], trigger and guide electric discharges [8], assist in the formation of raindrops and snow [9-11]. It emits characteristic fluorescence lines in the UV range, the predominant line of which at $337.1 \mathrm{~nm}$ is due to a transition between excited states $C^{3} \Pi_{u}^{+}$and $B^{3} \Pi_{g}^{+}$of the triplet manifold of neutral nitrogen molecules [12]. This fluorescence line has been commonly used to determine the length and width of filaments $[12,13]$, to estimate the filamentation laser intensity [14], or to remotely detect a $\mathrm{dc}$ or $\mathrm{THz}$ field $[15,16]$. Under pumping with powerful $(>7.5 \mathrm{~mJ})$ circularly polarized femtosecond pulses, the $337.1 \mathrm{~nm}$ line exhibits cavity-free lasing, both in the forward and backward direction with respect to the laser pulse [17,18]. The lasing in this case is interpreted in the following way. The free electrons generated with the circularly polarized pulse are initially distributed with a peak in their energy around $E=2 U_{p}$, where $U_{p}=e^{2} / c \varepsilon_{0} m_{e} \times I / 2 \omega_{0}^{2}$ is the ponderomotive energy [19].
If the pump pulse intensity exceeds $I \sim 10^{14} \mathrm{~W} / \mathrm{cm}^{2}$, the peak in the electron energy distribution is above the threshold $(\sim 11 \mathrm{eV})$ for impact excitation of ground state nitrogen molecules to the $C^{3} \Pi_{u}^{+}$level $[17,18,20]$. This leads to a fast transient population inversion between $C^{3} \Pi_{u}^{+}$and $B^{3} \Pi_{g}^{+}$. On the other hand, the origin of excitation of level $C^{3} \Pi_{u}^{+}$with linearly polarized pump light or circular pump with lower energy is still controversial. In the early literature, the following dissociative recombination scenario, $\mathrm{N}_{2}^{+}+\mathrm{N}_{2}+\mathrm{N}_{2} \rightarrow \mathrm{N}_{4}^{+}+\mathrm{N}_{2}$, followed by $\mathrm{N}_{4}^{+}+e \rightarrow$ $\mathrm{N}_{2}\left(C^{3} \Pi_{u}^{+}\right)+\mathrm{N}_{2}$ was proposed for the formation of nitrogen molecules in the $C^{3} \Pi_{u}^{+}$state $[12,21,22]$. This interpretation has been disputed by Arnold et al. who studied the line in a mixture gas of $\mathrm{N}_{2}$ and $\mathrm{He}$. Instead, they concluded that in their case the main mechanism was a collision-assisted intersystem crossing [23].

To distinguish between the various possible excitation transfer mechanisms from the plasma to the excited neutrals, it is useful to resort to time-resolved methods since different possible transfers occur with different rates [23]. Attempts have been made to measure the formation dynamics of $\mathrm{N}_{2}\left(C^{3} \Pi_{u}^{+}\right)$by time-resolved fluorescence with a time-gated photon multiplier tube or a streak camera [23-25]. However, the time resolution was limited to more than tens of picosecond or nanoseconds [23-25], which proved insufficient for this purpose.

In this Letter, we propose and demonstrate an all-optical method to monitor the formation of $\mathrm{N}_{2}\left(C^{3} \Pi_{u}^{+}\right)$, with a time 


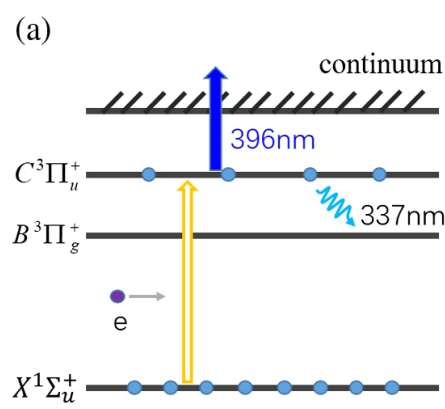

(b)

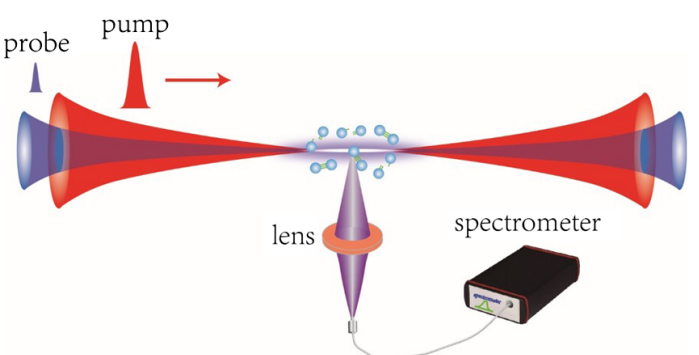

FIG. 1. (a) Energy levels of the nitrogen molecules and the principle of probe pulse $(396 \mathrm{~nm})$ induced fluorescence $(337.1 \mathrm{~nm})$ depletion. (b) The femtosecond pump and probe pulses are focused by a convex lens $(f=500 \mathrm{~mm})$ into a gas chamber filled with nitrogen gas or air at different pressures. The fluorescence from the air plasma filament is detected in the side with a spectrometer as a function of the pump-probe time delay $\tau$.

resolution of a few tens of femtoseconds (duration of the probe pulse). The principle of the experiment is the following: by injecting a short UV probe pulse inside the plasma filament, one observes a partial suppression of the emission at $337.1 \mathrm{~nm}$. This reduction of the fluorescence line is due to a transition from $C^{3} \Pi_{u}^{+}$state to the ionization continuum, as shown schematically in Fig. 1(a). By measuring the buildup of this fluorescence depletion as a function of pump-probe delay (following plasma formation at time $t_{0}$ ), one extracts directly the dynamics of formation of excited neutral nitrogen molecules, since the magnitude of the depression at time $t_{0}+\tau$ is directly proportional to the density of molecules in state $C^{3} \Pi_{u}^{+}$ at time $t_{0}+\tau$. We found that the formation time of excited neutral nitrogen molecules varies between $\sim 4$ ps at 1 bar gas pressure and $\sim 120 \mathrm{ps}$ at $30 \mathrm{mbar}$. This result leads us to the conclusion that electron impact excitation is the main channel for the formation of the excited neutral nitrogen molecules, while the dissociative recombination mechanism plays a negligible role. Our observations are well reproduced by rate equations.

The setup to implement the experiment is shown in Fig. 1(b). A commercial femtosecond laser system (Coherent Legend DUO) delivers 35 fs pulses at central wavelength of $796 \mathrm{~nm}$ with maximum pulse energy of $12 \mathrm{~mJ}$ at a repetition rate of $1 \mathrm{kHz}$. The output pulses are divided in two parts with a dielectric beam splitter. One pulse of $2 \mathrm{~mJ}$ serves as the main pump pulse to create the plasma filament. The second pulse passes through a type I BBO crystal to generate its second harmonics pulse around $396 \mathrm{~nm}$. The energy of the second harmonic pulses was measured to be $115 \mu \mathrm{J}$. The second harmonic photon energy, $h v=3 \mathrm{eV}$, is close to the energy difference $\Delta E=$ $4.3 \mathrm{eV}$ between $\mathrm{N}_{2}\left(C^{3} \Pi_{u}^{+}\right)$and the ionization continuum, and can therefore act as a probe pulse for fluorescence depletion via a two-photon transition. The two pulses are combined with a dichromatic mirror and focused by a convex lens $(f=500 \mathrm{~mm})$ into a gas chamber filled with pure nitrogen or air at different pressures. The time delay $\tau$ between the pump and probe pulses is varied with a motorized optical delay line. A bright plasma filament with length of $\sim 7 \mathrm{~mm}$ is formed in the middle of the gas chamber. The side fluorescence emitted by the plasma filament is first collimated by an $f=35 \mathrm{~mm}$ lens and then collected by another $f=100 \mathrm{~mm}$ lens into the fiber tip of a spectrometer.

In Fig. 2, we present the fluorescence spectrum of the filament plasma recorded with and without the probe pulse (red dotted and black lines) in pure nitrogen at 1 bar pressure. Lines at $337.1,357.8,380.5 \mathrm{~nm}$ all originate from the excited molecular level $\mathrm{N}_{2}\left(C^{3} \Pi_{u}^{+}\right)$. The quantum vibrational numbers of the upper and lower levels of each optical transition are denoted by the numbers in parenthesis. It is seen that upon injection of a delayed probe pulse

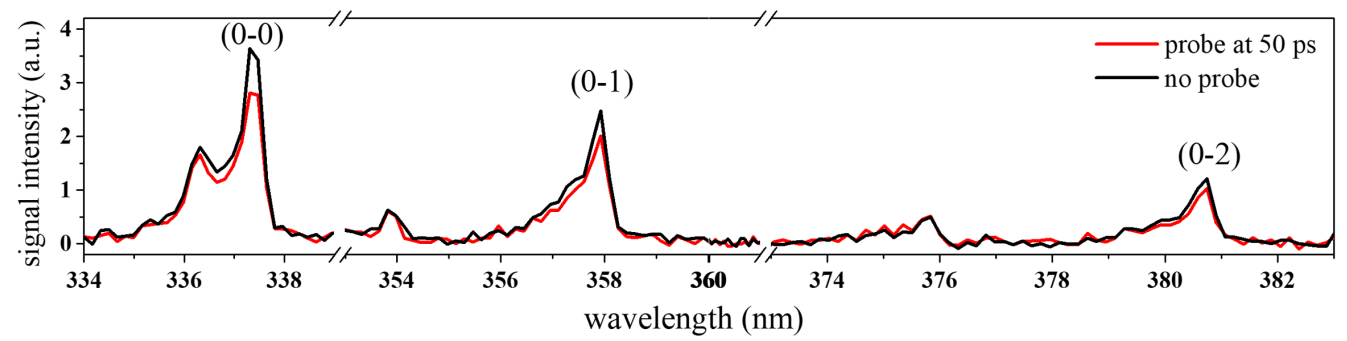

FIG. 2. Side fluorescence of the nitrogen gas plasma without (black line) and in the presence of (red line) of the probe pulse. The nitrogen pressure was 1 bar. 

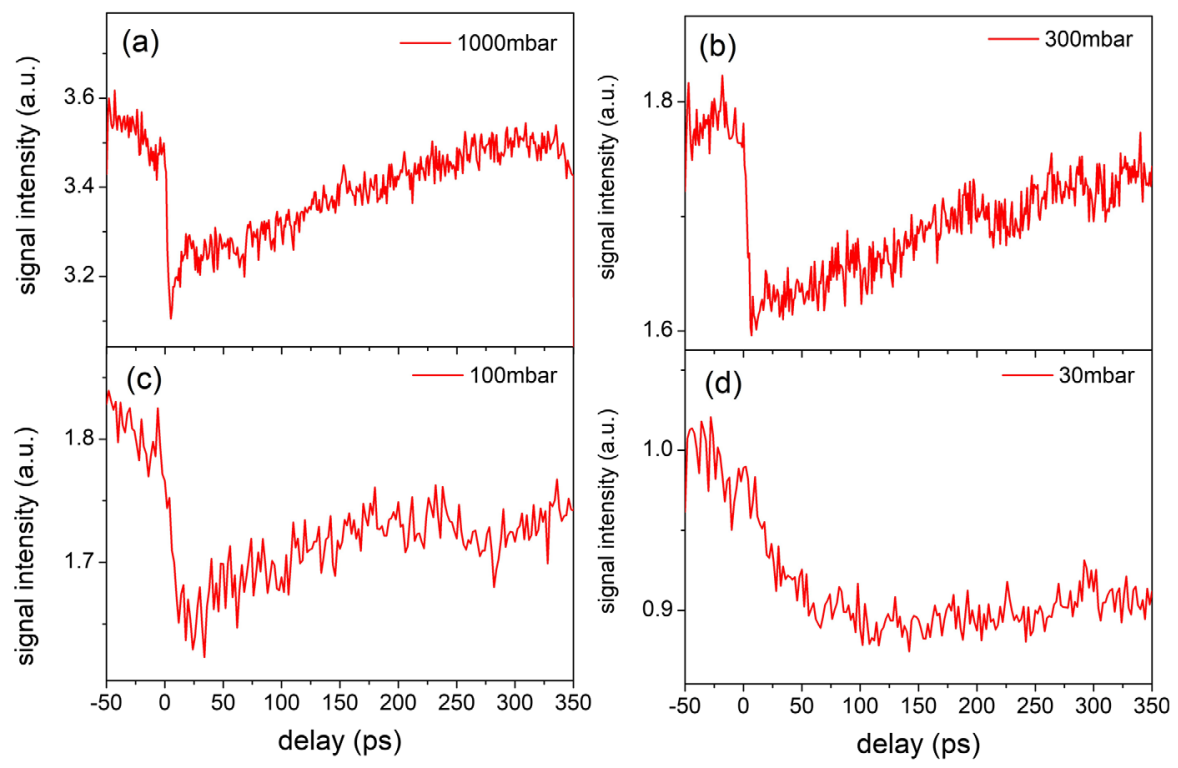

FIG. 3. Side fluorescence at $337.1 \mathrm{~nm}$ as a function of the pump-probe delay for different nitrogen gas pressures.

( $\tau=50 \mathrm{ps})$, a significant reduction of the emission intensity occurs for all lines $(337.1,357.8,380.5 \mathrm{~nm})$, corresponding to a decrease of the population of $\mathrm{N}_{2}\left(C^{3} \Pi_{u}^{+}\right)$.

We then scanned the pump-probe delay $\tau$ and recorded the corresponding fluorescence signal of line at $337.1 \mathrm{~nm}$, in order to monitor the formation dynamics of the $\mathrm{N}_{2}\left(C^{3} \Pi_{u}^{+}\right)$ molecules. The results are presented in Fig. 3 for different gas pressures. At atmospheric pressure, a rapid decrease of the fluorescence signal within a temporal range of $4 \pm 1 \mathrm{ps}$ is observed, corresponding to a formation time of $\mathrm{N}_{2}\left(C^{3} \Pi_{u}^{+}\right)$ in $4 \pm 1$ ps, as presented in Fig. 3(a). A scan with better temporal resolution is presented in Fig. 4(a). Upon further reduction of gas pressure, the $\mathrm{N}_{2}\left(C^{3} \Pi_{u}^{+}\right)$population buildup time becomes longer, as presented in Figs. 3(b)-3(d). For 30 mbar, it takes around $120 \mathrm{ps}$ for the population to reach a maximum.

We have tested the influence of pump laser polarization, with the experimental results presented in Fig. 4(a). In pure nitrogen at 1 bar pressure, it was found that the signal obtained with the linearly polarized pump laser is slightly larger than that with circular polarization, in agreement with a previous report in the lower intensity regime [20]. The decreasing behavior of the $337.1 \mathrm{~nm}$ signal is almost identical for both laser polarization states. Furthermore, we compared the results in pure nitrogen and air at different pressures, as shown in Fig. 4(b) and Fig. S1 of the Supplemental Material [26]. It is found that the signal presents a similar decreasing tendency, indicating that in air and pure nitrogen gas the $\mathrm{N}_{2}\left(C^{3} \Pi_{u}^{+}\right)$molecules formation dynamics are almost identical. Relying on the above systematic observations, we present in Fig. 5(a) the formation time of the $\mathrm{N}_{2}\left(C^{3} \Pi_{u}^{+}\right)$molecules as a function of gas pressure obtained in pure nitrogen gas with a linearly
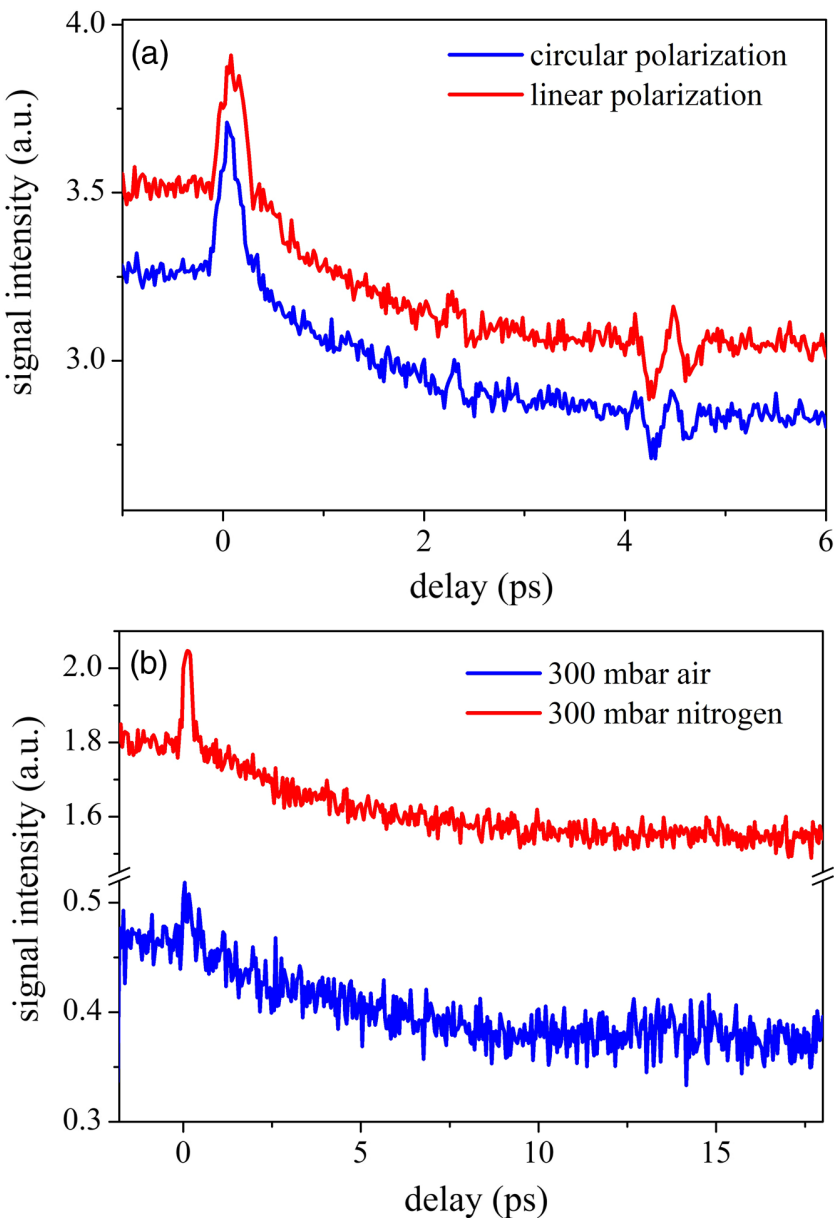

FIG. 4. Side $337.1 \mathrm{~nm}$ fluorescence as a function of the pumpprobe delay for different pump laser polarization (a), and comparison of air and pure nitrogen gas (b). In (a), the pressure of nitrogen was 1 bar. 

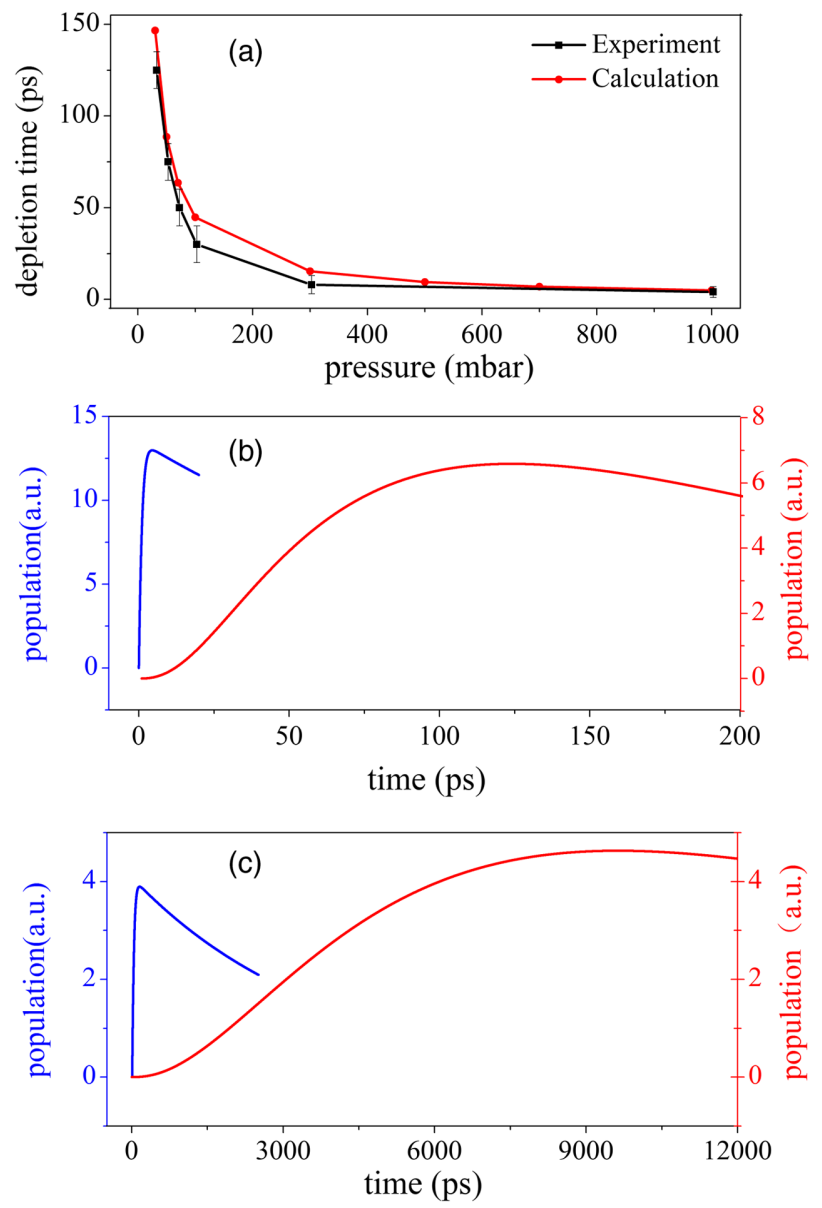

FIG. 5. (a) Experimentally measured (square dot) and calculated (solid circle) formation time of the $C^{3} \Pi_{u}^{+}$state molecules as a function of the nitrogen gas pressure. For 1 bar (b) and $30 \mathrm{mbar}$ (c) nitrogen, calculated formation dynamic of the $C^{3} \Pi_{u}^{+}$state molecules based on the electron impact mechanism (blue line) and the dissociative recombination mechanism (red line).

polarized pump pulse, and compare it with our numerical calculation described below.

How can we understand the fact that the excited $\mathrm{N}_{2}$ molecules are formed in less than 4 ps at atmospheric pressure? First, let us consider the dissociative recombination model. According to this model, the excited $\mathrm{N}_{2}$ molecules are the product of cascaded impact reactions between ionic and neutral nitrogen molecules. At 1 bar pressure, the mean collision time between nitrogen molecules can be estimated as $\tau_{c}=l / v_{\text {rms }}$, where $l$ is the free mean path and $v_{\text {rms }}$ is the average molecule speed at room temperature. With a mean free path $l=6.84 \times 10^{-8} \mathrm{~m}$ and average molecule speed $v_{\text {rms }}=284 \mathrm{~m} / \mathrm{s}$ at standard temperature and pressure (STP) conditions, the mean collision time is estimated to be $241 \mathrm{ps}$, significantly longer than the 4 ps characteristic time observed in Fig. 3(a). Therefore, we conclude that the dissociative recombination mechanism does not play a main role for $\mathrm{N}_{2}\left(C^{3} \Pi_{u}^{+}\right)$formation. An intersystem crossing scenario requires the presence of a high concentration of heavy atoms such as $\mathrm{He}$ with a resonant level for energy exchange, which is not the case in pure nitrogen or air. Now, we examine the mechanism of electron impact excitation of $\mathrm{N}_{2}$. In the STP condition, the mean collision time between electrons and nitrogen molecules can be estimated to be around 0.35 ps for electrons with energy of $11.1 \mathrm{eV}$, the threshold energy for electron collision excitation for the $C^{3} \Pi_{u}^{+}$state [27]. Therefore, in a temporal window of $4 \mathrm{ps}$, tens of inelastic collision events can occur, which can gradually populate level $\mathrm{N}_{2}\left(C^{3} \Pi_{u}^{+}\right)$ and qualitatively explain our observation $[28,29]$. For a more quantitative analysis, we simulate the formation dynamics of excited neutral molecules using a rate equation for the different neutral nitrogen excitation processes.

The rate equations that describe the dissociative recombination mechanism are the following:

$$
\begin{aligned}
\frac{d \mathrm{~N}_{2}\left(C^{3} \Pi_{u}^{+}\right)}{d t} & =k_{e p} n_{e} \mathrm{~N}_{4}^{+}-\frac{\mathrm{N}_{2}\left(C^{3} \Pi_{u}^{+}\right)}{\tau_{C}}-k_{q \mathrm{~N}_{2 C}} \mathrm{~N}_{2} \mathrm{~N}_{2}\left(C^{3} \Pi_{u}^{+}\right) \\
\frac{d \mathrm{~N}_{4}^{+}}{d t} & =k_{c} \mathrm{~N}_{2}^{+} \mathrm{N}_{2}-k_{e p} n_{e} \mathrm{~N}_{4}^{+} \\
\frac{d \mathrm{~N}_{2}^{+}}{d t} & =\alpha_{C I} n_{e}-k_{c} \mathrm{~N}_{2}^{+} \mathrm{N}_{2}-k_{e} n_{e} \mathrm{~N}_{2}^{+} .
\end{aligned}
$$

In these equations $k_{e p}=2 \times 10^{-6}\left[T_{e}(\mathrm{~K}) / 300\right]^{-0.5} \mathrm{~cm}^{3} / \mathrm{s}$ is the rate of electron capture by $\mathrm{N}_{4}^{+}, \quad k_{c}=6.8 \times$ $10^{-29}\left[300 / T_{v}(\mathrm{~K})\right]^{1.64} \mathrm{~N}_{2} \mathrm{~cm}^{3} / \mathrm{s}$ is the production rate of $\mathrm{N}_{4}^{+}, k_{e}=4.3 \times 10^{-8}\left(T_{e}[\mathrm{eV}]\right)^{-0.4} \mathrm{~cm}^{3} / \mathrm{s}$ is the rate of electron capture by $\mathrm{N}_{2}^{+}, \alpha_{C I}=7.6 \times 10^{11}\left\{\left[T_{e}(\mathrm{eV})\right] /[15.6]\right\}^{1.5} \times$ $\left\{[15.6] /\left[T_{e}(\mathrm{eV})\right]+2\right\} e^{-15.6 / T_{e}[\mathrm{eV}]} \mathrm{s}^{-1}$ is the ionization rate [30], $\tau_{C}=41.9 \times 10^{-9} \mathrm{~s}$ is the radiative lifetime, and $k_{q \mathrm{~N}_{2} C}=3 \times 10^{-10} \mathrm{~cm}^{3} / \mathrm{s}$ is the quenching rate of the triplet state through collisions with molecular nitrogen, as given in Ref. [30]. $T_{v}$ is the vibrational temperature.

The second mechanism, electron impact excitation, is described by the following equation:

$$
\begin{aligned}
\frac{d \mathrm{~N}_{2}\left(C^{3} \Pi_{u}^{+}\right)}{d t}= & \langle\sigma v\rangle n_{e} \mathrm{~N}_{2}-\frac{\mathrm{N}_{2}\left(C^{3} \Pi_{u}^{+}\right)}{\tau_{C}} \\
& -k_{q \mathrm{~N}_{2 C}} \mathrm{~N}_{2} \mathrm{~N}_{2}\left(C^{3} \Pi_{u}^{+}\right) .
\end{aligned}
$$

The cross section $\sigma$ is obtained in analytical form from Ref. [31]. In our model, we assume a Maxwellian distribution for the velocity of the electrons to compute $\langle\sigma v\rangle[30,32]$.

The electron density and temperature of the plasma electrons is computed using a OD model, described in Refs. [30,32]:

$$
\begin{aligned}
\frac{d n_{e}}{d t} & =\alpha_{C I} n_{e}+k_{e p} \mathrm{~N}_{4}^{+} n_{e}-k_{e} \mathrm{~N}_{2}^{+} n_{e}-\eta n_{e}, \\
\frac{3}{2} \frac{d n_{e} T_{e}}{d t} & =-Q_{C} \mathrm{~N}_{2} n_{e}\left(1-\frac{T_{v}}{T_{e}}\right), \\
\frac{3}{2} \mathrm{~N}_{2} \frac{d T_{v}}{d t} & =Q_{C} \mathrm{~N}_{2} n_{e}\left(1-\frac{T_{v}}{T_{e}}\right) .
\end{aligned}
$$


In these equations the attachment rate is $\eta=2.75 \times$ $10^{-10} T_{e}^{-0.5} e^{-5 / T_{e}} \mathrm{~N}_{2}+1.5 \times 10^{-32} T_{e}^{-1} e^{-0.052 / T_{e}} \mathrm{~N}_{2}^{2} \mathrm{~s}^{-1}$ and the cooling rate is $Q_{C}=3.5 \times 10^{-8} e^{-5 / 3 T_{e}}+6.2 \times$ $10^{-11} e^{-13 T_{e}}$ for $0.1 \mathrm{eV}<T_{e}<2 \mathrm{eV}[30,32]$. For higher temperatures, we interpolate linearly the logarithm of $Q_{C}$. The plasma model is coupled with the molecular path or the electronic path model to study the different mechanisms.

We now examine the formation dynamics of the $C^{3} \Pi_{u}^{+}$ state population based on the above two mechanisms. For $1 \mathrm{bar}$ and 30 mbar nitrogen gas, the calculation results are shown in Figs. 5(b) and 5(c). In Fig. 5(b), the formation time is determined to be $\sim 4 \mathrm{ps}$ according to the electron impact mechanism, in good agreement with the experimental observation. In contrast, when only the molecular path is considered, it is around 125 ps (red line), much longer than the experimental measurement. This also agrees with our qualitative analysis. We have calculated the formation time of the $C^{3} \Pi_{u}^{+}$state molecules for different gas pressures. The results are presented in Fig. 5(a). Reasonable agreement is found between calculation and experiment for all pressures ranging from 30 mbar to 1 bar. Numerical simulations also revealed that the electron energy distribution function after photoionization is not crucial for the formation dynamic of the $C^{3} \Pi_{u}^{+}$ state molecules. This explains the almost identical temporal dynamics observed for linearly and circularly polarized pump pulses as presented in Fig. 4(a).

In conclusion, we have revealed the temporal formation dynamics of the excited $\mathrm{N}_{2}$ molecules inside air plasma filaments generated by femtosecond laser pulses, based on a laser induced fluorescence depletion method. It was found that the excited $\mathrm{N}_{2}$ molecules are formed in less than 4 ps in 1 bar nitrogen gas. With reduced gas pressure, this formation time increases up to $120 \mathrm{ps}$ for air pressure of 30 mbar. This ultrafast formation process cannot be explained by the previously proposed dissociative recombination mechanism, or the intersystem crossing scenario. Our numerical simulation based on the electron impact excitation mechanism reproduce quantitatively this ultrafast formation dynamic and it dependence on air pressure. We therefore conclude that the excited neutral nitrogen molecules inside filamentary plasma occurs mainly via electron impact excitation. This clarifies a long-standing fundamental problem and can be important for the applications of filaments based on the excited neutral nitrogen molecules such as remote detection of electric fields, cavity-less air lasers, and filament characterization.

The work is supported in part by the National Natural Science Foundation of China (Grants No. 11574213 and No. 11904332), Innovation Program of Shanghai Municipal Education Commission (Grant No. 2017-0107-00-07-E00007), and Shanghai Municipal Science and Technology Commission (No. 17060502500). Y. L. acknowledges the support by The Program for Professor of Special Appointment (Eastern Scholar) at Shanghai Institutions of Higher Learning (No. TP2014046). E. O. acknowledges the support by the Programa Propio de $\mathrm{I}+\mathrm{D}+\mathrm{i}$ of the Universidad Politécnica de Madrid, project DERKETA.

R. D. and X. Z. contributed equally to this work.

*eduardo.oliva@upm.es

†yi.liu@usst.edu.cn

[1] A. Couairon and A. Mysyrowicz, Femtosecond filamentation in transparent media, Phys. Rep. 441, 47 (2007).

[2] S. L. Chin, S. A. Hosseini, W. Liu, Q. Luo, F. Théberge, N. Aközbek, A. Becker, V. P. Kandidov, O. G. Kosareva, and H. Schroeder, The propagation of powerful femtosecond laser pulses in optical media: Physics, applications, and new challenges, Can. J. Phys. 83, 863 (2005).

[3] F. Martin, R. Mawassi, F. Vidal, I. Gallimberti, D. Comtois, H. Pépin, J. C. Kieffer, and H. P. Mercure, Spectroscopic study of ultrashort pulse laser-breakdown plasmas in air, Appl. Spectrosc. 56, 1444 (2002).

[4] F. Théberge, W. Liu, P. Tr. Simard, A. Becker, and S. L. Chin, Plasma density inside a femtosecond laser filament in air: Strong dependence on external focusing, Phys. Rev. E 74, 036406 (2006).

[5] C. D'Amico, A. Houard, M. Franco, B. Prade, A. Mysyrowicz, A. Couairon, and V. T. Tikhonchuk, Conical Forward THz Emission from Femtosecond Laser Filamentation in Air, Phys. Rev. Lett. 98, 235002 (2007).

[6] K. Y. Kim, A. J. Taylor, J. H. Glownia, and G. Rodriguez, Coherent control of terahertz supercontinuum generation in ultrafast laser-gas interactions, Nat. Photonics 2, 605 (2008).

[7] Y. Brelet, A. Houard, G. Point, B. Prade, L. Arantchouk, J. Carbonnel, Y.-B. André, M. Pellet, and A. Mysyrowicz, Radiofrequency plasma antenna generated by femtosecond laser filaments in air, Appl. Phys. Lett. 101, 264106 (2012).

[8] J. Kasparian et al., White-light filaments for atmospheric analysis, Science 61, 301 (2003).

[9] P. Rohwetter et al., Laser-induced water condensation in air, Nat. Photonics 4, 451 (2010).

[10] J. Ju, J. Liu, C. Wang, H. Sun, W. Wang, X. Ge, C. Li, S. L. Chin, R. Li, and Z. Xu, Laser-filamentation-induced condensation and snow formation, Opt. Lett. 37, 1214 (2012).

[11] J. Ju et al., Temporal evolution of condensation and precipitation induced by a 22-TW laser, Opt. Express 26, 2785 (2018).

[12] A. Talebpour, S. Petit, and S. L. Chin, Re-focusing during the propagation of a focused femtosecond Ti: Sapphire laser pulse in air, Opt. Commun. 171, 285 (1999).

[13] H. Yang, J. Zhang, W. Yu, Y. J. Li, and Z. Y. Wei, Long plasma channel generated by femtosecond laser pusles, Phys. Rev. E 65, 016406 (2001).

[14] S. Xu, X. Sun, B. Zeng, W. Chu, J. Zhao, W. Liu, Y. Cheng, Z. $\mathrm{Xu}$, and S. L. Chin, Simple method of measuring laser peak intensity inside femtosecond laser filament in air, Opt. Express 20, 299 (2012).

[15] K. Sugiyama, T. Fujii, M. Miki, M. Yamaguchi, A. Zhidkov, E. Hotta, and K. Nemoto, Laser-filament-induced corona 
discharges and remote measurements of electric fields, Opt. Lett. 34, 2964 (2009).

[16] J. Liu, J. Dai, S. L. Chin, and X.-C. Zhang, Broadband terahertz wave remote sensing using coherent manipulation of fluorescence from asymmetrically ionized gases, Nat. Photonics 4, 627 (2010).

[17] S. Mitryukovskiy, Y. Liu, P. Ding, A. Houard, and A. Mysyrowicz, Backward stimulated radiation from filaments in nitrogen gas and air pumped by circularly polarized $800 \mathrm{~nm}$ femtosecond laser pulses, Opt. Express 22, 12750 (2014).

[18] P. Ding, E. Oliva, A. Houard, A. Mysyrowicz, and Y. Liu, Lasing dynamics of neutral nitrogen molecules in femtosecond filaments, Phys. Rev. A 94, 043824 (2016).

[19] P. B. Corkum, N. H. Burnett, and F. Brunel, AboveThreshold Ionization in the Long-Wavelength Limit, Phys. Rev. Lett. 62, 1259 (1989).

[20] S. Mitryukovskiy, Y. Liu, P. Ding, A. Houard, A. Couairon, and A. Mysyrowicz, Plasma Luminescence from Femtosecond Filaments in Air: Evidence for Impact Excitation with Circularly Polarized Light Pulses, Phys. Rev. Lett. 114, 063003 (2015).

[21] H. L. Xu, A. Azarm, J. Bernhardt, Y. Kamali, and S. L. Chin, The mechanism of nitrogen fluorescence inside a femtosecond laser filament in air, Chem. Phys. 360, 171 (2009).

[22] H. L. Xu, A. Azarm, and S. L. Chin, Controlling fluorescence from $\mathrm{N}_{2}$ inside femtosecond laser filaments in air by two-color laser pulses, Appl. Phys. Lett. 98, 141111 (2011).

[23] B. R. Arnold, S. Roberson, and P. M. Pellgrino, Excited state dynamics of nitrogen reactive intermediates at the threshold of laser induced filamentation, Chem. Phys. 405, 9 (2012).

[24] P. Wang, S. Xu, D. Li, H. Yang, H. Jiang, Q. Gong, and C. Wu, Spectroscopic study of laser-induced tunneling ionization of nitrogen molecules, Phys. Rev. A 90, 033407 (2014).

[25] M. W. Lei, C. Wu, Q. Liang, A. Zhang, Y. Li, Q. Cheng, S. Wang, H. Yang, Q. Gong, and H. Jiang, The fast decay of ionized nitrogen molecules in laser filamentation investigated by a picosecond streak camera, J. Phys. B 50, 145101 (2017).

[26] See Supplemental Material at http://link.aps.org/ supplemental/10.1103/PhysRevLett.123.243203 for fluorescence depletion as a function of pump-probe delay in air and nitrogen at different pressures.

[27] M. Danko, J. Orszagh, A. Ribar, and S. Matejcik, Electron impact excitation of the second positive system of $\mathrm{N}_{2}$, in 12th International Symposium on High Pressure Low Temperature Plasma Chemistry, Bratislava, Slovakia, 2010.

[28] D. Kartashov, S. Ališauskas, A. Pugžlys, M. Shneider, and A. Baltuška, Theory of a filament initiated nitrogen laser, J. Phys. B 48, 094016 (2015).

[29] D. Kartashov and M. N. Shneider, Femtosecond filament initiated, microwave heated cavity-free nitrogen laser in air, J. Appl. Phys. 121, 113303 (2017).

[30] J. Peñano, P. Sprangle, B. Hafizi, D. Gordon, R. Fernsler, and M. Scully, Remote lasing in air by recombination and electron impact excitation of molecular nitrogen, J. Appl. Phys. 111, 033105 (2012).

[31] T. Tabata, T. Shirai, M. Sataka, and H. Kubo, Analytic cross sections for electron impact collisions with nitrogen molecules, At. Data Nucl. Data Tables 92, 375 (2006).

[32] P. Sprangle, J. Peñano, B. Hafizi, D. Gordon, and M. Scully, Remotely induced atmospheric lasing, Appl. Phys. Lett. 98, 211102 (2011). 